\title{
Physical inactivity is not the same as sedentarism: the harm of prolonged sitting to human health
}

\begin{abstract}
In this opinion paper I have tried to compel convincing evidence for the harmful effects of physical inactivity, and how prolonged sitting time favours skeletal muscle metabolic inflexibility through its connection with the development of non-communicable diseases, such as obesity, diabetes, hypertension, cardiovascular diseases, among others. It is my point of view that there is already enough evidence regarding the deleterious effects of physical inactivity on human health. Therefore, it is imperative that physical activity must be included in post-modern human as a daily habit in order to fight against noncommunicable diseases.
\end{abstract}

Volume 3 Issue 5 - 2017

\author{
Viviane LA Nouailhetas \\ Department of Biophysics, Federal University of São Paulo, \\ Brazil
}

Correspondence: Viviane LA Nouailhetas, Department of Biophysics, Federal University of São Paulo, Brazil,

Email vivi.nouailhetas@gmail.com

Received: May 29, 2017 | Published: June 05, 2017

\section{Opinion}

Post-modern life style in great western cities, as a consequence of the dramatic scientific and technological development, imposed profound alterations in individual daily life behavior as compared to hunter-gathering or agricultural society's life style. Nowadays, enhancement of daily activity automation, associated with the general transportation trends, and predominant use of digital machines by the whole population both at work and leisure times dramatically reduced daily energy expenditure by promoting gradual increments in our sitting, non-standing time. In addition, recent diet quality also decreased through the dramatic introduction of industrialized food, and lack of fruits and vegetables, greatly facilitating adipose tissue deposit. Coincidently, epidemiological evidence shows that there is a serious and dramatic onslaught of the so called non-communicable diseases (NCD). Those include obesity, diabetes, hypertension, coronary heart disease, intestinal diseases, some types of cancer, Alzheimer disease and Parkinson disease, and depression. ${ }^{1-4}$ Some of them, such as obesity diabetes, and hypertension, seem to be linked to skeletal muscle metabolic inflexibility. ${ }^{5}$ This latter is characterized by impairment in the choice of the right energetic substrate according to skeletal muscle motor activity, thus causing alteration in glucose (greater fast blood glucose) and lipid metabolism (increased total cholesterol, triglycerides), ectopic lipid accumulation, and systemic pre-inflammatory. Environment (increased circulating C-reactive protein), a condition known as metabolic syndrome. ${ }^{6}$ Indeed there is now general consensus and reasonable epidemiological data which show that these diseases seem to decrease life expectancy. Additionally, it is connected to important deterioration of life quality in elder lies, which represents a growing fraction of the world population, consequently enhancing social and financial cost to public health.

The explosion of $\mathrm{NCD}^{1,3}$ is quite surprising, considering the knowledge accumulated in the last 20years concerning beneficial effects of structured exercise to human health. Nowadays physicists do prescribe habitual and moderate aerobic exercise as the best nonpharmacological strategy to keep human body homeostasis. Indeed, it improves cardiovascular and pulmonary conditioning and developing a favorable blood lipid profile, in contrast to resistance exercise which enhances skeletal muscular strength and mass (hypertrophy). Corroborating the systemic effect of exercise we showed that habitual and chronic moderate treadmill exercise improves cognition, protects intestine, cardiac and skeletal muscle from the deleterious effects of aging, while chronic moderate (up to 55days) or intense exercise (10days) cause dramatic changes in intestinal morphology and signaling transduction mechanisms related or not to oxidative stress in rodent animal model. ${ }^{7-11}$ In addition, exercise-induced redox biology signaling has been proposed to modulate glycemic control and skeletal muscle adaptation to exercise, thus leading to important metabolic modifications. ${ }^{12}$

Sedentarism behavior, on the other hand, is attributed to absence of the recommended minimum weekly structured exercise to promote good health status, and is considered the inferior end-point of the exercise continuum. However, sedentarism is now claimed to have distinct molecular, cellular, physiological, and clinical features from those underlying physical inactivity. The contribution of the non-exercise physical activity thermogenesis (NEAT) to total daily energetic expenditure might constitute a higher fraction in comparison to structured exercise activity. ${ }^{13}$ Accordingly, NEAT is defined as all energy expenditure associated with spontaneous physical activity, and is quite variable among individuals since it is connected to complex interaction of environmental and biological factors related to people diverse occupation and leisure-time activities. NEAT reaches its lower level when daily activity involves prolonged periods of sitting time (higher than $4 \mathrm{~h}$ ) during work- and/or leisure-time activities). Indeed, one of the most critical differences is the cellular regulation of skeletal muscle lipoprotein lipase, an important enzyme for controlling plasma triglyceride catabolism, HDL cholesterol, and other metabolic risk factors. Evidence shows that this enzyme is up-regulated by structured exercise, but dramatically down-regulated by physical inactivity. ${ }^{14}$ In that sense it is very attractive the elegant hypothesis proposed by Booth and colleagues ${ }^{2,15}$ that physical inactivity might be related to the expression of a set of "physical inactivity genes" which favors metabolic fluxes that promote increased fat adipose tissue, skeletal muscle metabolic inflexibility, mild systemic inflammation, and skeletal muscle atrophy. In addition, the increased prevalence of chronic diseases in the post-modern society is believed to be primarily due to absence of the minimum level of physical activity which associated with inadequate high energetic diet favor the expression of the "physical inactivity" pattern of the human genome, thus leading to development of chronic diseases. 
It is my point of view that there is already enough evidence regarding the deleterious effects of physical inactivity on human health. Therefore, it is imperative that physical activity must be included in post-modern human as a daily habit in order to fight against non-communicable diseases. My proposal is that this new physical inactivity paradigm, even though not well-established yet, should be disseminated among the population, and that health professionals and educators act accordingly to convince population (mainly the children and youth) to avoid prolonged periods of sitting both at occupational and/or leisure-times.

\section{Acknowledgements}

None.

\section{Conflict of interest}

Author declares that there is no conflict of interest.

\section{References}

1. Lee IM, Shiroma EJ, Lobelo F, et al. Effect of physical inactivity on major non-communicable diseases worldwide: an analysis of burden of disease and life expectancy. Lancet. 2012;380(9838):219-229.

2. Booth FW, Chakravarthy MV, Gordon SE, et al. Waging war on physical inactivity: using modern molecular ammunition against an ancient enemy. J Appl Physiol. 2002;93(1):3-30.

3. Booth FW, Gordon SE, Carlson CJ, et al. Waging war on modern chronic diseases: primary prevention through exercise biology. $J \mathrm{Appl}$ Physiol. 2000;88(2):774-787.

4. Ekelund U, Steene Johannessen J, Brown WJ, et al. Does physical activity attenuate, or even eliminate, the detrimental association of sitting time with mortality? A harmonised meta-analysis of data from more than 1million men and women. Lancet. 2016;388(10051):1302-1310.
5. Rynders CA, Blanc S, DeJong N, et al. Sedentary behavior is a key determinant of metabolic inflexibility. $J$ Physiol. 2018;596(8):1319-1330.

6. Febraio MA. Exercise and inflammation. $J$ Appl Physiol. 2007;103(1):376-377.

7. de Lira CA, Vancini RL, Ihara SS, et al. Aerobic exercise affects C57BL/6 murine intestinal contractile function. Eur J Appl Physiol. 2008;103(2):215-223.

8. Rosa EF, Alves GA, Luz J, et al. Activation of HPA axis and remodeling of body chemical composition in response to an intense and exhaustive exercise in C57BL/6 mice. Physiol Res. 2014;63(5):605-613.

9. Rosa EF, Freymüller E, Ihara SS, et al. Damaging effects of intense repetitive treadmill running on murine intestinal musculature. $J \mathrm{Appl}$ Physiol. 2008;104(5):1410-1417.

10. Rosa EF, Silva AC, Ihara SS, et al. Habitual exercise program protects murine intestinal, skeletal, and cardiac muscles against aging. $J$ Appl Physiol. 2005;99(4):1569-1575.

11. Rosa EF, Takahashi S, Aboulafia J, et al. Oxidative stress induced by intense and exhaustive exercise impairs murine cognitive function. $J$ Neurophysiol. 2007;98(3):1820-1826.

12. Parker L, Shaw CS, Stepto NK, et al. Exercise and glycemic control: focus on redox homeostasis and redox-sensitive protein signaling. Front Endocrinol (Lausanne). 2017;8:87.

13. Hamilton MT, Hamilton DG, Zderic TW. Role of low energy expenditure and sitting in obesity, metabolic syndrome, type 2 diabetes, and cardiovascular disease. Diabetes. 2007;56(11):2655-2667.

14. Zderic TW, Hamilton MT. Physical inactivity amplifies the sensitivity of skeletal muscle to the lipid-induced down regulation of lipoprotein lipase activity. J Appl Physiol. 2006;100(1):249-257.

15. Booth FW, Roberts CK, Matthew JL. Lack of exercise is a major cause of chronic diseases. Compr Physiol. 2012;2(2):1143-1211. 